\title{
Developments in the Management of Advanced Non Small Cell Lung Cancer
}

\author{
Alexandra Kopitopoulou ${ }^{1}$, Ioannis Ntanos $^{1}$, Muhammad W. Saif ${ }^{2}$, Kostas Syrigos ${ }^{3 *}$ \\ ${ }^{1}$ Department of Pulmonology, "Sotiria" General Hospital, Athens, Greece; ${ }^{2}$ Experimental Therapeutics, Tufts University School of \\ Medicine, Boston, USA; ${ }^{3}$ Oncology Unit GPP, “Sotiria” General Hospital, Athens School of Medicine, Athens, Greece. \\ Email: *ksyrigos@med.uoa.gr
}

Received November $12^{\text {th }}, 2012$; revised December $13^{\text {th }}, 2012$; accepted December $21^{\text {st }}, 2012$

\begin{abstract}
Background: Overall survival (OS) and quality of life in patients with advanced non small cell lung cancer (NSCLC) remains to the day highly unsatisfactory. Better understanding of the mechanisms of oncogenesis resulted in the development of new drugs, which target a specific stage in tumor development. Additionally, optimal timing of chemotherapy administration has been readdressed with the introduction of maintenance treatment in clinical practice. The aim of this review is to briefly summarize the developments in first line treatment of NSCLC with small molecule tyrosine kinase inhibitors, the role of crizotinib and the current options in maintenance treatment. Methodology: A search was performed in the PubMed database and in Clinical Trials.gov using the keywords erlotinib, gefitinib, crizotinib and maintenance treatment. Findings were reported in form of a narrative review. Conclusions: First line treatment with small molecule TKIs for NSCLC is both effective and feasible. EGFR mutation status remains the main prognostic factor of response. Pemetrexed and Erlotinib, currently approved for Maintenance Treatment in NSCLC, seem to benefit patients with advanced disease. Identification of suitable candidates for Maintenance Treatment, optimal timing and agent selection are issues that still remain largely unanswered. Early study results promise that addition of crizotinib to the treatment regimen of ALK positive NSLCL is likely to be beneficiary for a substantial number of patients.
\end{abstract}

Keywords: Erlotinib; Gefitinib; Crizotinib; Maintenance Treatment; NSCLC

\section{Introduction}

Overall survival (OS) and quality of life in patients with advanced non small cell lung cancer (NSCLC) remains to the day highly unsatisfactory. Until recently, the only therapeutic choice for these patients was standard platinum doublet chemotherapy. Cisplatin or Carboplatin in combination with Taxanes, Vinca Alkaloids or Antimetabolites represented the cornerstone of treatment. The urgent need for more effective agents and the better understanding of the mechanisms of oncogenesis resulted in the development of new drugs, which target a specific stage in tumor development. Additionally, optimal timing of chemotherapy administration has been readdressed with the introduction of maintenance treatment in clinical practice.

\section{NSCLC First Line Treatment with Small Molecule Tyrosine Kinase Inhibitors (TKIs)}

Treatment of NSCLC has changed significantly since Tyrosine Kinase Inhibitors-TKIs were instituted in clinical practice. The discovery of EGFR (Epidermal

*Corresponding author.
Growth Factor Receptor) mutations in a subgroup of patients with NSCLC in 2004 lead to the introduction of targeted treatment in thoracic oncology, treatment depending on the genetic profile of the disease [1].

EGFR belongs to the transmembrane HER/ErbB receptor family and mediates the transduction of several extracellular ligands, as are the Epidermal Growth Factor (EGF), the Transforming Growth Factor alpha (TGF- $\alpha$ ) and amphiregulin. EGFR is over expressed in numerous malignancies and thus it was used as a target for the development of new antineoplastic agents. Erlotinib (Tarceva) and Gefitinib (Iressa) are small molecule TKIs which selectively inhibit the phosphorylization of the intracellular EGFR kinase domain. They are administered orally and have a mild toxicity profile, the main dose-limiting toxicities being diarrhea and skin rash [2].

Gefitinib has been approved for use on patients already treated for NSCLC, with the phase II study IDEAL-2 (Iressa Dose Evaluation in Advanced Lung Cancer 2). Erlotinib has been approved for the same indication with the phase III trial BR. 21, which demonstrated better response rates in patients of Asian origin, women, never or light smokers and patients with a histo- 
logical pattern of adenocarcinoma. It was then revealed that these subgroups of patients harbored mutations of the EGFR gene, which were not present in Gefitinib and Erlotinib resistant tumors and are referred to as TKIsensitizing EGFR mutations.

The first studies for the use of TKIs as first line agents produced disappointing results. They were performed on patients with NSCLC, without controlling for TKI sensitizing EGFR mutations. Seven completed phase II studies were retrieved. A phase II trial of Erlotinib showed a response rate of $23 \%$ with a median progression-free survival (PFS) of 2.8 months [3] and found that response rate was linked to histological type and smoking status. Two trials showed a response rate of $5 \%$ and $15 \%$ in unselected patients treated with first line Gefitinib [4,5]. Two other single-arm studies of Erlotinib, one in elderly patients and one in patients with low performance status (PS $>2$ ), demonstrated response rates of $8 \%$ and $10 \%$ respectively [6,7]. In the phase II Iressa Versus Vinorelbine trial (INVITE) [8], previously untreated elderly patients were randomized to Gefitinibor Vinorelbine, and the response rate for Gefitinib was 3\%. There was no statistically significant difference found between the two treatments. Another trial compared Erlotinib to combination Carboplatin and Paclitaxel inpatients with a PS $\geq 2$. It showed a 4\% response rate for Erlotinib and a better overall survival in the chemotherapy arm of 9.5 months [9]. The TOPICAL trial (Erlotinib in Treating Patients with Stage III or Stage IV Non Small Cell Lung Cancer, clinical trials. gov identifier NCT00275132) is a randomized phase II trial of Erlotinib as first line treatment in unselected patients not fit to receive chemotherapy. The study was completed in March 2012 and results are anticipated soon.

Other phase II studies for the use of TKIs as first line treatment in NSCLC included patients with specific clinical characteristics. Two studies which were performed on Asians, a population which is known to have high rates of EGFR mutations. These studies showed response rates of 30\% [10] and 51\% [11]. Another Japanese study of Gefitinib in elderly patients, [12] showed a response rate of $25 \%$. A study by Lee et al. evaluated Gefitinib in Asian patients with adenocarcinoma who were neversmokers and showed a response rate of $33 \%$ with a median overall survival of 20 months [13]. A study of Erlotinib which included women with adenocarcinoma who were never-smokers or former smokers showed a response rate of $30 \%$ [14]. In the same study, patients who were positive for EGFR mutations had response rates of $70 \%$.

A study of first-line Erlotinib in patients with predominantly BAC type adenocarcinoma [15] showed a response rate of $21 \%$. Two other studies of Gefitinib in BAC type adenocarcinoma [16,17] have found response rates of $13 \%$ and $17 \%$. Mucinous BAC is usually KRAS positive, which is a negative predictive factor of response to EGFR TKIs. Non-mucinous BAC has a better response than mucinous BAC to Gefitinib.

Studies that were performed on populations with molecular confirmation of EGFR mutations present with the best results. In the ONCOBELL trial of Gefitinib in advanced NSCLC, patients were controlled for EGFR mutation status by fluorescence in situ hybridization (FISH) and immunohistochemistry (IHC) with P-Akt staining [18]. Never smokers were eligible even if only one marker was positive or if no tissue was available. The response rate was $57 \%$ in patients positive for EGFR IHC, $68 \%$ inpatients positive for EGFRFISH, and $71 \%$ in patients with TKI-sensitizing EGFR mutations.

Two studies of first line Gefitinib performed on Japanese population positive for EGFR mutations $[19,20]$ showed a response rate of $75 \%$. Another study took place in the United States and evaluated Gefitinib inpatients with non-squamous cell carcinoma positive for EGFR mutations, finding a response rate of 55\% [21].

A large multicenter Spanish study which included only patients with EGFR mutations was published in 2009 [22]. Approximately two thousand lung cancer patients were screened for EGFR mutations and 350 patients were found positive. The study, a phase II trial of firstline Erlotinib included 217 of these patients. Persons not previously treated had a response rate of $74 \%$, with a median progression-free survival of 14 months and median overall survival of 28 months.

A Japanese study evaluated first-line Gefitinib in patients with EGFR mutations who were not fit to receive chemotherapy due to poor PS [23]. Inclusion criteria were a sensitizing mutation in EGFR exon 19 or 21 , as well as age specific poor PS. The response rate was $66 \%$ and median overall survival was 17.8 months. $68 \%$ of patients had an improvement of PS from $3-4$ to $0-1$, a phenomenon described as "Lazarus response".

The IPASS study (Iressa Pan-Asia Study) was a randomized controlled phase III trial which compared first line treatment with TKIs to classical chemotherapy with a platinum doublet. It took place in Asia and included patients who were never smokers or former light smokers, had not received previous treatment and had adenocarcinoma. The participants were randomized to receive Gefitinibor Carboplatin/Paclitaxel combination and the primary endpoint was PFS. The trial proved that Gefitinib was superior to the platinum doublet in response rate and PFS [24]. 85\% of the patients additionally consented to EGFR mutation analysis. In patients with EGFR mutation the response rate was $71 \%$ and the PFS10 months versus $1 \%$ and less than 2 months respectively in patients with no mutation.

In the EURTAC trial, (European Randomized Trial of 
Tarceva versus Chemotherapy) patients were randomized to receive Erlotinib as first line treatment or classical chemotherapy with a platinum doublet. An independent data monitoring committee recommended that the phase III EURTAC study be stopped early because it met its primary endpoint. It was shown that, compared to platinum-based chemotherapy, Erlotinib significantly extended PFS in newly diagnosed advanced NSCLC with TKIsensitizing EGFR mutations.

In conclusion, TKIs should be administered as first line treatment in patients with EGFR mutation positive NSCLC [25]. Testing for these mutations in patients with adenocarcinoma should become routine clinical practice [26].

\section{Maintenance Treatment}

Maintenance Treatment in NSCLC is administered to patients who show no disease progression after four to six cycles of initial chemotherapy. Maintenance Treatment includes both Continuation and Switch Maintenance. According to the National Comprehensive Cancer Network guidelines, Continuation Maintenance refers to the use of at least one of the agents used in first line treatment. Switch Maintenance refers to the initiation of a different agent, which was not included in the first line regimen.

In the Continuation Maintenance setting, which includes continuation with the non platinum agent due to toxicity, several trials are available. These trials are the continuation of Paclitaxel (Belani, 2003), Gemcitabine (Brodowicz, 2006, Belani, 2010 and Perol, 2010), and Pemetrexed (Paz Arez 2011) [27]. They randomized patients with no progression after four cycles of chemotherapy (or 16 weeks in the Paclitaxel trial) to continue with the non platinum agent until progression. With Continuation Maintenance, there are positive results regarding PFS with both Gemcitabine and Pemetrexed. None of these studies demonstrates a benefit in overall survival. Thus, Continuation Maintenance with these agents cannot be recommended as standard practice. However, it is known that if Bevacizumab and Cetuximab are used in the first line setting they should be continued until progression, as is demonstrated by the licensing studies for these agents (ECOG 4599, AVAIL and FLEX respectively).

In Switch Maintenance, patients who have no progression after four cycles of first line platinum doublet chemotherapy are randomized to receive immediate treatment with one of the four agents used in second line treatment, versus standard treatment at disease progression. These trials include switch to Vinorelbine (Westeel et al., 2005), Docetaxel (Fidias et al., 2009), PemetrexedJMEN trial (Ciuleanu et al., 2009), Erlotinib-SATURN
(Capuzzo, 2010) and ICFT trial (Perol, 2010), GefitinibEORTC trial (Gafaar et al., 2010) and to Erlotinib with Bevacizumab-ATLAS trial (Miller 2009) [27]. Allstudies show a benefit in PFS but only the JMEN and the SATURN study managed to show an advantage in overall survival. The JMEN trial of Pemetrexed resulted in a median overall survival benefit of 13.4 versus 10.6 months. However, patients with squamous cell cancers, who had no benefit from Pemetrexed, were also included in the study. A subset analysis of the patients with non squamous cancers showed an improved overall survival benefit of 5.2 months. The SATURN study of Erlotinib Switch Maintenance Therapy showed a benefit in median overall survival of one month, from 11 to 12 months. The trial demonstrates that patients with stable disease after first line treatment benefit more from maintenance Erlotinib (with a 2.3 month improvement in overall survival) than patients who present radiological response after first line treatment.

A meta-analysis by Behera and colleagues [27] also concluded that Switch maintenance was associated with a significant benefit in both overall and progression free survival. On the other hand, Continuation Maintenance was associated only with progression free survival benefit and showed no impact on overall survival.

There is debate as to whether the benefit seen in the switch maintenance trials is a result of chemotherapy timing (i.e. offering it early after 4 cycles of initial chemotherapy versus offering it on progression) or whether it is the consequence of more patients receiving further active treatment. This is especially relevant as even within the setting of clinical trials less than $50 \%$ of patients receive second line therapy on progression.

According to data from these studies, patients who should receive maintenance treatment are those who have the worst prognostic factors and have benefited less from first line treatment. Indeed, patients who will fail to show improvement will probably have to proceed soon to alternative chemotherapy. Unfortunately, the trials in Maintenance Treatment did not estimate the effect of prognostic factors of response as are histological type, EGFR mutation or response to first line treatment. In practice, in patients positive for EGFR mutation Erlotinib should be preferred. Patients with wild type EGFR and non-squamous histology should receive Pemetrexed. It is at this point safe to say that Maintenance treatment offers these patients a chance of better survival through earlier treatment, before further treatment becomes impossible due to disease progression.

\section{Crizotinib}

Recently, an oncogenic rearrangement of the Anaplastic Lymphoma Kinase has been discovered in NSCLC (ALK, Anaplastic Lymphoma Kinase). ALK is an oncogene and 
in lung cancer it is activated through fusion with the EML-4 oncogene. It is estimated that $2 \%-7 \%$ of NSCLC is positive for the ALK mutation [28].

Crizotinibis an oral ATP-competitive selective inhibittor of the ALK and MET tyrosine kinase, which inhibits tyrosine phosphorylation of activated ALK at nanomolar concentrations. Dose escalation, toxic effects, and pharmacokinetics were reported in 2009 [29]. Toxicity profile is mild, with mainly gastrointestinal side effects.

An early trial of Crizotinib in patients with ALK positive advanced NSCLC by Kwak et al. showed a response rate of $57 \%$, with an estimated probability of six-month progression-free survival of $72 \%$ [30]. In a retrospective study by Shaw et al., the investigators compared survival outcomes in Crizotinib treated patients and Crizotinib naïve controls [31]. Both groups included ALK positive and ALK negative cancers. The study concluded that in patients with advanced NSCLC, ALK-positive, Crizotinib therapy is associated with improved survival. It was also shown that ALK rearrangement is not a favorable prognostic factor in advanced NSCLC.

The PROFILE 1005 study is a phase IItrial which included ALK positive NSCLC patients with disease progression after previous treatment [32]. The early results show that Crizotinib is safe and well-tolerated with preliminary evidence of improved symptoms and clinically meaningful antitumor activity in patients with pre-treated ALK-rearranged NSCLC.

To the date a number of Crizotinibtrials for NSCLC are in progress, with early results expected in about a year (NCT00965731, NCT01500824, NCT00932893, NCT00932451, NCT01154140, NCT01441128).

\section{Conclusions}

First line treatment with small molecule TKIs for NSCLC is both effective and feasible, with mild toxicity profile and oral administration route being key advantages of these agents. Careful patient selection according to EGFR mutation status is detrimental, as this remains the main prognostic factor of response.

Pemetrexed and Erlotinib, the drugs currently approved for Maintenance Treatment in NSCLC, seem to benefit patients with advanced disease. Identification of suitable candidates for Maintenance Treatment, optimal timing and agent selection are issues that still remain largely unanswered. Toxicity from prolonged treatment should always be taken into account and weighed against the expected benefits.

Crizotinib is a new weapon in the fight against cancer. As with other TKIs, the mild toxicities and easy administration are favorable attributes. Promising results with this agent to the day, lead us to hope that its addition to the treatment regimen of ALK positive NSLCL will be beneficiary for a substantial number of patients.
After decades of stagnant water in the treatment of advanced NSCLC, new treatment opportunities are emerging. The discovery of new mutations, the development of targeted agents and new treatment strategies are slowly moving this disease into a new light. With a long road ahead, the way is finally beginning to look promising.

\section{REFERENCES}

[1] E. A. Kotteas, A. G. Charpidou and K. N. Syrigos, "Targeted Therapy for Nonsmall Cell Lung Cancer: Focusing on Angiogenesis, the Epidermal Growth Factor Receptor and Multikinase Inhibitors," Anticancer Drugs, Vol. 21, No. 2, 2010, pp. 151-168.

doi:10.1097/CAD.0b013e328334da02

[2] A. Charpidou, D. Blatza, V. Anagnostou and K. N. Syrigos, "EGFR Mutations in Non-Small-Cell Lung Cancer-Clinical Implications," In Vivo, Vol. 22, No. 4, 2008, pp. 529-536.

[3] G. Giaccone, M. Gallegos Ruiz, T. Le Chevalier, et al., "Erlotinib for Frontline Treatment of Advanced NonSmall-Cell Lung Cancer: A Phase II Study," Clinical Cancer Research, Vol. 12, No. 20, 2006, pp. 6049-6055. doi:10.1158/1078-0432.CCR-06-0260

[4] M. Reck, E. Buchholz, K. S. Romer, et al., "Gefitinib Monotherapy in Chemotherapy-Naive Patients with Inoperable Stage III/IV Non-Smallcell Lung Cancer," Clin Lung Cancer, Vol. 7, No. 6, 2006, pp. 406-411. doi:10.3816/CLC.2006.n.025

[5] W. Akerley, K. M. Boucher, J. S. Bentz, et al., "A Phase II Study of Erlotinib as Initial Treatment for Patients with Stage IIIB-IV Non-Small-Cell Lung Cancer," Journal of Thoracic Oncology, Vol. 4, No. 2, 2009, pp. 214-219. doi:10.1097/JTO.0b013e3181943bb9

[6] D. M. Jackman, B. Y. Yeap, N. I. Lindeman, et al., "Phase II Clinical Trial of Chemotherapy-Naive Patients $>$ or $=70$ Years of Age Treated with Erlotinib for Advanced Non-Small-Cell Lung Cancer," Journal of Clinical Oncology, Vol. 25, No. 7, 2007, pp. 760-766. doi:10.1200/JCO.2006.07.5754

[7] P. J. Hesketh, K. Chansky, A. J. Wozniak, et al., "Southwest Oncology Group Phase II Trial (S0341) of Erlotinib (OSI-774) in Patients with Advanced Non-Small-Cell Lung Cancer and a Performance Status of 2," Journal of Thoracic Oncology, Vol. 3, No. 9, 2008, pp. 1026-1031. doi:10.1097/JTO.0b013e318183aa1f

[8] L. Crino, F. Cappuzzo, P. Zatloukal, et al., "Gefitinib versus Vinorelbine in Chemotherapy-Naive Elderly Patients with Advanced Non-Small-Cell Lung Cancer (INVITE): A Randomized, Phase II Study," Journal of Clinical Oncology, Vol. 26, No. 26, 2008, pp. 4253-4260. doi:10.1200/JCO.2007.15.0672

[9] R. Lilenbaum, R. Axelrod, S. Thomas, et al., "Randomized Phase II Trial of Erlotinib or Standard Chemotherapy in Patients with Advanced Non-Small-Cell Lung Cancer and a Performance Status of 2," Journal of Clinical Oncology, Vol. 26, No. 6, 2008, pp. 863-869.

doi:10.1200/JCO.2007.13.2720 
[10] S. Niho, K. Kubota, K. Goto, et al., "First-Line Single Agent Treatment with Gefitinib in Patients with Advanced Non-Small-Cell Lung Cancer: A Phase II Study," Journal of Clinical Oncology, Vol. 24, No. 1, 2006, pp. 64-69. doi:10.1200/JCO.2005.02.5825

[11] C. H. Yang, C. J. Yu, J. Y. Shih, et al., "Specific EGFR Mutations Predict Treatment Outcome of Stage IIIB/IV Patients with Chemotherapy-Naive Non-Small-Cell Lung Cancer Receiving First-Line Gefitinib Monotherapy," Journal of Clinical Oncology, Vol. 26, No. 16, 2008, pp. 2745-2753. doi:10.1200/JCO.2007.15.6695

[12] N. Ebi, H. Semba, S. J. Tokunaga, et al., "A Phase II Trial of Gefitinib Monotherapy in Chemotherapy-Naive Patients of 75 Years or Older with Advanced Non-SmallCell Lung Cancer," Journal of Thoracic Oncology, Vol. 3, No. 10, 2008, pp. 1166-1171. doi:10.1097/JTO.0b013e318186a88d

[13] D. H. Lee, J. Y. Han, S. Y. Yu, et al., "The Role of Gefitinib Treatment for Korean Never-Smokers with Advanced or Metastatic Adenocarcinoma of the Lung: A Prospective Study," Journal of Thoracic Oncology, Vol. 1, No. 9, 2006, pp. 965-971. doi:10.1097/01243894-200611000-00008

[14] D. M. Jackman, L. Cioffredi, N. I. Lindeman, et al., "Phase II Trial of Erlotinib in Chemotherapy-Naive Women with Advanced Pulmonary Adenocarcinoma (Abstract 8065)," Journal of Clinical Oncology, Vol. 27, No. 15S, 2009, p. 423s.

[15] V. A. Miller, G. J. Riely, M. F. Zakowski, et al., "Molecular Characteristics of Bronchioloalveolar Carcinoma and Adenocarcinoma, Bronchioloalveolar Carcinoma Subtype, Predict Response to Erlotinib," Journal of Clinical Oncology, Vol. 26, No. 9, 2008, pp. 1472-1478. doi:10.1200/JCO.2007.13.0062

[16] H. L. West, W. A. Franklin, J. McCoy, et al., "Gefitinib Therapy in Advanced Bronchioloalveolar Carcinoma: Southwest Oncology Group Study S0126," Journal of Clinical Oncology, Vol. 24, No. 12, 2006, pp. 1807-1813. doi:10.1200/JCO.2005.04.9890

[17] J. Cadranel, E. Quoix, L. Baudrin, et al., "IFCT-0401 trial: A Phase II Study of Gefitinib Administered as First-Line Treatment in Advanced Adenocarcinoma with Bronchioloalveolar Carcinoma Subtype," Journal of Thoracic Oncology, Vol. 4, No. 9, 2009, pp. 1126-1135. doi:10.1097/JTO.0b013e3181abeb5d

[18] F. Cappuzzo, C. Ligorio, P. A. Janne, et al., "Prospective Study of Gefitinib in Epidermal Growth Factor Receptor Fluorescence in Situ Hybridization-Positive/PhosphoAkt-Positive or Never Smoker Patients with Advanced Non-Small-Cell Lung Cancer: The ONCOBELL Trial," Journal of Clinical Oncology, Vol. 25, No. 16, 2007, pp. 2248-2255. doi:10.1200/JCO.2006.09.4300

[19] H. Asahina, K. Yamazaki, I. Kinoshita, et al., "A Phase II Trial of Gefitinib as First-Line Therapy for Advanced Non-Small-Cell Lung Cancer with Epidermal Growth Factor Receptor Mutations," British Journal of Cancer, Vol. 95, No. 8, 2006, pp. 998-1004. doi:10.1038/sj.bjc. 6603393

[20] A. Inoue, T. Suzuki, T. Fukuhara, et al., "Prospective
Phase II Study of Gefitinib for Chemotherapy-Naive Patients with Advanced Non-Small-Cell Lung Cancer with Epidermal Growth Factor Receptor Gene Mutations," Journal of Clinical Oncology, Vol. 24, No. 21, 2006, pp. 3340-3346. doi:10.1200/JCO.2005.05.4692

[21] L. V. Sequist, R. G. Martins, D. Spigel, et al., "First-Line Gefitinib in Patients with Advanced Non-Small-Cell Lung Cancer Harboring Somatic EGFR Mutations," Journal of Clinical Oncology, Vol. 26, No. 15, 2008, pp. 2442-2449. doi:10.1200/JCO.2007.14.8494

[22] R. Rosell, T. Moran, C. Queralt, et al., "Screening for Epidermal Growth Factor Receptor Mutations in Lung Cancer," The New England Journal of Medicine, Vol. 361, No. 10, 2009, pp. 958-967. doi:10.1056/NEJMoa0904554

[23] A. Inoue, K. Kobayashi, K. Usui, et al., "First-Line Gefitinib for Patients with Advanced Non-Small-Cell Lung Cancer Harboring Epidermal Growth Factor Receptor Mutations without Indication for Chemotherapy," Journal of Clinical Oncology, Vol. 27, No. 9, 2009, pp. 13941400. doi:10.1200/JCO.2008.18.7658

[24] T. S. Mok, Y.-L. Wu, S. Thongprasert, et al., "Gefitinib or Carboplatin-Paclitaxel in Pulmonary Adenocarcinoma," The New England Journal of Medicine, Vol. 361, No. 10, 2009, pp. 947-957. doi:10.1056/NEJMoa0810699

[25] I. Gkiozos, A. Charpidou and K. Syrigos, "Developments in the Treatment of Non-Small-Cell Lung Cancer," Anticancer Research, Vol. 27, No. 4C, 2007, pp. 2823-2827.

[26] G. S. Papaetis, C. Roussos and K. N. Syrigos, "Targeted Therapies for Non-Small-Cell Lung Cancer," Current Pharmaceutical Design, Vol. 13, No. 27, 2007, pp. 28102831. doi:10.2174/138161207781757079

[27] A. G. Pallis and K. Syrigos, "Targeted (and Chemotherapeutic) Agents as Maintenance Treatment in Patients with Metastatic Non-Small-Cell Lung Cancer: Current Status and Future Challenges," Cancer Treatment Reviews, Vol. 38, No. 7, 2012, pp. 861-867. doi:10.1016/j.ctrv.2011.12.006

[28] G. Mountzios, D. Sanoudou and K. N. Syrigos, "Clinical Pharmacogenetics in Oncology: The Paradigm of Molecular Targeted Therapies," Current Pharmaceutical Design, Vol. 16, No. 20, 2010, pp. 2184-2193. doi:10.2174/138161210791792859

[29] E. L. Kwak, D. R. Camidge, J. Clark, et al., "Clinical Activity Observed in a Phase I Dose Escalation Trial of An Oral C-MET and ALK Inhibitor, PF-02341066," Journal of Clinical Oncology, Vol. 27, No. 15S, 2009, p. $148 \mathrm{~s}$.

[30] E. Kwak, Y. Bang, D. Camidge, et al., "Anaplastic Lymphoma Kinase Inhibition in Non-Small-Cell Lung Cancer," The New England Journal of Medicine, Vol. 363, No. 18, 2010, pp. 1693-1703.

[31] A. Shaw, B. Yeap, B. Solomon, et al., "Effect of Crizotinib on Overall Survival in Patients with Advanced NonSmall-Cell Lung Cancer Harboring ALK Gene Rearrangement: A Retrospective Analysis," The Lancet Oncology, 2011, Vol. 12, No. 11, pp. 1004-1012. doi:10.1016/S1470-2045(11)70232-7 
[32] L. Crinò, D. Kim, G. J. Riely, et al., "Initial Phase II Results with Crizotinib in Advanced ALK-Positive NonSmall Cell Lung Cancer (NSCLC): PROFILE 1005,"
Journal of Clinical Oncology, Vol. 29, No. 15, 2011, p. 7514. 